\title{
Based on HPA axis to explore the mechanism of waist-rubbing therapy in the treatment of lumbar disc herniation model rats
}

\section{Yuhang Qin ( 26qyh221@163.com )}

Second Clinical Medical college of Nanjing University of TCM

\section{Facai Lin}

Acupuncture and Tuina college of Nanjing University of TCM

\section{Yunchuan Wu}

Acupuncture and Tuina College of Nanjing University of TCM

\section{Ying Xiong}

Acupuncture and Tuina College of Nanjing University of TCM

\section{Guanghan Sun}

First Clinical Medical College of Anhui University of Traditional Chinese Medicine

\section{Research}

Keywords: lumbar disc herniation, waist-rubbing method, HPA axis, GR, kidney nourishing

Posted Date: May 3rd, 2020

DOI: https://doi.org/10.21203/rs.3.rs-20031/v1

License: (c) (i) This work is licensed under a Creative Commons Attribution 4.0 International License. Read Full License 


\section{Abstract}

\section{Background}

lumbar disc herniation (LDH) is a high-risk species in the world with serious harm. Traditional Chinese Tuina is the first choice in the treatment of LDH. In the past clinical studies, we have achieved good results in the treatment of LDH with the waist-rubbing method recorded in an ancient Chinese medical book of Qing Dynasty. Now we further explore its internal mechanism of action from the perspective of animal experiments. Waist-rubbing is a frictional heat-generating stimulus that acts on the surface of the skin. It does not relieve the compression in spinal canal, but can effectively eliminate the symptoms of LDH patients. This suggests that waist-rubbing may play a therapeutic role by interfering the autoimmunity of LDH. For a long time, the treatment of LDH mainly focused on how to relieve the mechanical compression caused by lumbar disc herniation, but little attention was paid to the autoimmune inflammation of LDH. In this study, from the point of view of regulating LDH's autoimmunity, the waist-rubbing method was taken as the treatment measure, and the lumbar strain rats in upright position were taken as the model. The neuroendocrine immunology mechanism of waist-rubbing method in treating LDH was revealed, which provided new ideas and scientific basis for Tuina clinical treatment of LDH, and also provided strong evidence for the theory of LDH's autoimmunity.

\section{Methods}

30 SD rats were randomly divided into three groups of the blank, the model and the waist wiping. The LDH model of the forelimb-free standing rats was established. Each group was treated according to the corresponding method. The waist-rubbing group was treated once a day for 15 days. After the experiment, the blood and lumbar spinal cord samples were collected after rats been killed. The contents of the $\mathrm{CRH}$ (Corticotropin releasing hormone), ACTH(Adrenocorticotropic hormone), CORT(Cortisol), IL17(Interleukin-17), IL-6(Interleukin-6), TGF- $\beta_{1}$ (Transforming growth factor- $\beta_{1}$ ) and IL-10(Interleukin-10) in peripheral blood and the $\mathrm{GR}_{\mathrm{a}}$ (Glucocorticoid receptor $\mathrm{a}$ ) and $5-\mathrm{HT}_{1 \mathrm{~A}}$ (Five hydroxytryptamine $1 \mathrm{~A}$ ) receptor in L4-L6 segment of rat lumbar spinal cord were detected by ELISA(Enzyme-linked immunoSorbent assay).

\section{Results}

Compared with the blank group, the contents of $\mathrm{CRH}, \mathrm{ACTH}, \mathrm{CORT}, \mathrm{GR}, \mathrm{a}-\mathrm{HT} \mathrm{TA}_{1 \mathrm{~A}}$ receptor, TGF- $\beta_{1}$ and IL-10 in the model group were significantly decreased $(P<0.05)$, while the contents of IL-17 and IL- 6 were significantly increased $(P<0.05)$. Compared with the model group, the contents of $\mathrm{CRH}, \mathrm{ACTH}, \mathrm{CORT}, \mathrm{GR}_{\mathrm{a}}$ $5-\mathrm{HT}_{1 \mathrm{~A}}$ receptor, TGF- $\beta_{1}$ and IL-10 in the waist-rubbing group were significantly increased $(P<0.05)$, while the contents of IL-17 and IL-6 were significantly decreased $(P<0.05)$. 


\section{Conclusion}

The waist-rubbing method may eliminate the autoimmune inflammatory reaction of LDH by correcting the hypofunction of HPA axis, so as to achieve the therapeutic effect of LDH.

\section{Background}

LDH is a kind of clinical syndrome, which is caused by the herniated lumbar disc that stimulating or compressing the adjacent spinal nerve roots, resulting in the main symptoms of waist pain and/or lower limbs numbness pain. It has a high incidence and serious harm. Tuina, as a Chinese traditional medical technology for $\mathrm{LDH}$, has a significant effect and widely used ${ }^{1}$. Our previous clinical observation has proved that the waist-rubbing method can effectively relieve the lumbocrural pain of patients ${ }^{2}$. Now we further explore the molecular mechanism of its treatment of LDH from the perspective of adjusting the hypothalamic-pituitary-adrenal axis (HPA axis), so as to provide more scientific basis for the clinical treatment of Tuina.

\section{Materials And Methods}

\section{Experimental animal}

30 adult Sprague-Dawley rats, male and female unlimited, weighed about 250-310 g, purchased from Shanghai Experimental Animal Center (License No. SCXK(Shanghai) 2016-0001). The rats were fed according to the requirements of the cleaning level and ate freely.

The main experimental drugs and apparatus

The main experimental drugs were the ELISA kits of CRH, ACTH, CORT, IL-17, IL-6, TGF- $\beta_{1}, \mathrm{IL}-10$, GR $\mathrm{R}_{\mathrm{a}}$ and $5-\mathrm{HT}_{1 \mathrm{~A}}$ receptor (All of the above kits were purchased from Nanjing Jiancheng biology Co., Ltd.). The main experimental apparatus were Micro CT machine (SCONCE, Switzerland), electric homogenizer (FLUKO, Germany), pipette (Eppendorf, Germany), constant temperature incubator (Shanghai Yiheng Scientific Instrument Co., Ltd.), full-automatic enzyme labeling instrument (Beijing PLANON New Technology Co., Ltd.), low-temperature high-speed centrifuge (Hettich, Germany).

Grouping and modeling

30 rats were divided into three groups: blank group, model group and waist-rubbing group according to the random number table, 10 rats in each group. Except for the blank group, the other two groups were made into the model of forelimb-free standing rats according to Liu Dun's LDH modeling method ${ }^{3}$, the operation was as follows:

Rats were anesthetized by intraperitoneal injection of $10 \%$ chloral hydrate at a weight of $0.5 \mathrm{ml} / 100 \mathrm{~g}$. Aftar chavina and alnanina hath fanrlimhe of rats, the forelimb skin was disinfected with iodophor. Cut Loading [MathJax]/jax/output/CommonHTML/jax.js 
the skin laterally at the proximal $1 / 3$ of the forelimb, peeled off the fascia and muscles, exposed the deltoid vascular nerve bundles, and ligated with threads. At the distal end of the ligation site, the humerus was severed with bone forceps, and then the skin, muscle, blood vessel and nerve were cut with scissors, so that the upper limbs were severed. After gentamicin was dripped into the incision, the muscle, fascia, and skin were sutured layer by layer. Finally, chlortetracycline eye ointment was rubbed on the incision to prevent infection. After the operation, the model rats were fed in normal cage for 2 weeks until wound recovered, then they were fed in special cage. The height of special cage was higher than that of common cage, and the height of food tank and water bottle could be adjusted at will. The height of food tank and water bottle was adjusted according to the average value which was measured every week from the vertical height of rats. The rats obtained food and water by standing upright, so as to maintain the continuous longitudinal pressure of lumbar vertebrae to promote the formation of LDH. The rats were kept in special cage for 12 weeks until the model was formed (It took 14 weeks to complete the modeling).

Model identification and rejection were required after modeling: $\otimes$ Rats appeared limping and dragging gait. $\otimes$ The lower lumbar scan of the model rats was performed with a micro-CT machine to confirm that there was a disc herniation between any two segments of L4-S1.

If the model rats meet the above two conditions at the same time, it was considered that the model was successfully included in the study, and the rest were excluded.

Intervention scheme

Treatment was performed 1 week after confirming successful LDH modeling. Treatment was given once a day for 15 consecutive days.

The blank group and the model group were not given any treatment.

Waist-rubbing group was given the following operation:

The rats were fixed safely. The wrist joint of the operator was extended and maintained a certain tension, and the index and middle fingers were closed and pasted lightly on the lumbosacral part of the rat. Under the joint flexion and extension movement of shoulder joint and elbow joint, the index and middle fingers were driven to make an evenly straight-line back-and-forth friction movement. The rubbing was continued for $1 \mathrm{~min}$ at a frequency of 80 times/min.

Sample collection and index detection

Sample collection

After the experiment, all of rats were anesthetized with $10 \%$ chloral hydrate and killed after blood collection from abdominal aorta. Collected $5 \mathrm{ml}$ of blood, leaved it at room temperature for $2 \mathrm{~h}$, centrifunated it at $30 n \mathrm{n} / \mathrm{min}$ for $15 \mathrm{~min}$ then took the supernatant and stored it at $-20^{\circ} \mathrm{C}$. The $\mathrm{L}_{4}-\mathrm{L}_{6}$ Loading [MathJax]/jax/output/CommonHTML/jax.js 
spinal cord was cut, then put into container with lysate added. The tissue homogenate was centrifuged at $12000 \mathrm{r} / \mathrm{min}$ for $20 \mathrm{~min}$, then the supernatant of homogenate was taken and stored at $-20^{\circ} \mathrm{C}$.

Index detection

The contents of CRH, ACTH, CORT, IL-17, IL-6, TGF- $\beta_{1}$, IL-10 in serum and $\mathrm{GR}_{a}, 5-\mathrm{HT}_{1 \mathrm{~A}}$ receptor in spinal cord were detected by ELISA. The experimental procedures were strictly in accordance with the instructions of these kits.

Data processing

The obtained data were analyzed by SPSS 17.0 statistical software for analysis of variance $L S D$ - $t$ test. It was set that $\mathrm{a}=0.05, P<0.05$, which was considered statistically significant.

\section{Results}

Sample shedding situation

In the model group, 2 substandard ones were removed after the model identification. In the waist-rubbing group, all of them passed the evaluation, but one died in the middle of the experiment, which was also eliminated. The blank samples were kept normally.

Comparison of serum hormone content

The contents of $\mathrm{CRH}, \mathrm{ACTH}$ and CORT in the model group were significantly lower than those in the blank group $(P<0.05)$. The $\mathrm{CRH}$ content in the waist-rubbing group was not statistically significant compared with the blank group $(P>0.05)$, while the ACTH and CORT contents were significantly increased $(P<$ 0.05).Compared with the model group, the above hormone indexes of the waist-rubbing group were significantly higher $(P<0.05)$. (Table 1$)$

Table 1

\begin{tabular}{|lllll|}
\multicolumn{5}{|c|}{ Comparison of serum CRH, ACTH and CORT $(\mathrm{pg} / \mathrm{mL}, \bar{x} \pm s)$} \\
\hline Group & $\mathbf{n}$ & CRH & ACTH & CORT \\
\hline Blank group & 10 & $25.16 \pm 3.26$ & $37.33 \pm 1.58$ & $136.12 \pm 10.51$ \\
\hline Model group & 8 & $18.44 \pm 2.85^{\#}$ & $20.15 \pm 2.37^{\#}$ & $95.46 \pm 9.33^{\#}$ \\
\hline W-R group & 9 & $26.87 \pm 3.01 \triangle \star$ & $40.32 \pm 1.64^{\# \star}$ & $148.31 \pm 11.26^{\# \star}$ \\
\hline Note: $\otimes$ Compared with the blank group: $P>0.05^{\triangle}, P<0.05^{\#}$ \\
\hline Q Compared with the model group: $P<0.05^{\star}$ \\
\hline
\end{tabular}


Compared with the blank group, the levels of L-17 and IL-6 in the serum of the model group were significantly increased $(P<0.05)$, while the levels of TGF- $\beta_{1}$ and IL-10 were significantly reduced $(P<$ 0.05). The levels of IL- 6 and TGF- $\beta_{1}$ in the serum of the waist-rubbing group were not statistically significant compared with the blank group $(P>0.05)$, while the levels of IL-17 and IL-10 were significantly different $(P<0.05)$. Compared with the model group, the levels of L-17 and IL-6 in the serum of the waistrubbing group were significantly reduced $(P<0.05)$, while the levels of TGF- $\beta_{1}$ and IL-10 were significantly increased $(P<0.05)$. (Table 2)

\section{Table 2}

Comparison of serum inflammatory factors $(\mathrm{ng} / \mathrm{L}, \bar{X} \pm s)$

\begin{tabular}{|c|c|c|c|c|c|}
\hline Group & n & L-17 & IL-6 & TGF- $\beta_{1}$ & IL-10 \\
\hline Blank group & 10 & $23.19 \pm 2.43$ & $10.46 \pm 2.38$ & $120.83 \pm 12.91$ & $25.67 \pm 2.45$ \\
\hline Model group & 8 & $45.81 \pm 3.27^{\#}$ & $21.07 \pm 2.56^{\#}$ & $92.40 \pm 8.35^{\#}$ & $18.55 \pm 3.42^{\#}$ \\
\hline W-R group & 9 & $25.33 \pm 1.35^{\#_{\star}}$ & $12.15 \pm 2.47 \triangle \star$ & $127.56 \pm 11.24 \triangle *$ & $29.73 \pm 2.13^{\# \star}$ \\
\hline \multicolumn{6}{|c|}{ Note: $\otimes$ Compared with the blank group: $P>0.05^{\triangle}, P<0.05^{\#}$} \\
\hline \multicolumn{6}{|c|}{$\otimes$ Compared with the model group: $P<0.05^{\star}$} \\
\hline
\end{tabular}

Comparison of $\mathrm{GR}_{\mathrm{a}}$ and $5-\mathrm{HT}_{1 \mathrm{~A}}$ receptor contents in spinal cord

The contents of $\mathrm{GR}_{\mathrm{a}}$ and $5-\mathrm{HT}_{1 \mathrm{~A}}$ receptor in the spinal cord of the model group were significantly lower than those of the blank group $(P<0.05)$. The content of $\mathrm{GR}_{\mathrm{a}}$ in the spinal cord of the waist-rubbing group was not statistically significant compared with the blank group $(P>0.05)$, while the content of $5-\mathrm{HT}_{1 \mathrm{~A}}$ receptor was significantly different $(P<0.05)$. Compared with the model group, the contents of $\mathrm{GR}_{\mathrm{a}}$ and 5 $\mathrm{HT}_{1 \mathrm{~A}}$ receptor in the spinal cord of the waist-rubbing group were significantly increased $(P<0.05)$. (Table 3) 
Table 3

Comparison of $\mathrm{GR}_{\mathrm{a}}$ and $5-\mathrm{HT}_{1 \mathrm{~A}}$ receptor contents in spinal $\operatorname{cord}(\mathrm{pg} / \mathrm{mL}, \bar{X} \pm s)$

\begin{tabular}{|lcll|}
\hline Group & $\mathbf{n}$ & $\mathbf{G R}_{\mathbf{a}}$ & $\mathbf{5 - H T}_{1 \mathrm{~A}}$ receptor \\
\hline Blank group & 10 & $85.37 \pm 11.28$ & $10.33 \pm 1.25$ \\
\hline Model group & 8 & $46.15 \pm 9.57^{\#}$ & $6.39 \pm 2.06^{\#}$ \\
\hline W-R group & 9 & $82.71 \pm 10.13^{\triangle \star}$ & $12.27 \pm 2.11^{\# \star}$ \\
\hline Note: $\otimes$ Compared with the blank group: $P>0.05^{\triangle}, P<0.05^{\#}$ \\
\hline \Compared with the model group: $P<0.05^{\star}$ \\
\hline
\end{tabular}

\section{Discussion}

Since Naylor ${ }^{4}$ and others proposed LDH's autoimmune theory, there have been continuous studies to prove that there is indeed a sustained immune inflammatory response induced by the herniated nucleus pulposus in the spinal canal of LDH patients. It is this sustained autoimmune response caused patients with long-term and repeated lumbago and leg pain. However, for many years, most of the related reports only focus on the study of changes in immune complements and antibodies in the cerebrospinal fluid. It has even not yet made deep research progress on the mechanism of LDH immune disorders.

The production of various life activities of the human body cannot be separated from the regulation of the nervous system, so the formation of autoimmunity must also be affected by the nervous system. The HPA axis is the main outgoing pathway for the nervous system to regulate the immune system. It participates in regulating the function of the immune system by secreting neurotransmitters and a variety of endocrine hormones. The immune system also reacts to HPA axis through a variety of cytokines and hormone-like substances. The two systems maintain the stability of the internal environment through the interaction mechanism. Therefore, it is a new path to explore the formation mechanism of LDH autoimmunity from the function change of HPA axis.

$\mathrm{CRH}, \mathrm{ACTH}$, and CORT are representative hormones secreted by the HPA axis, which can effectively reflect the functional status of the HPA axis. Glucocorticoid (GC), mainly CORT, is the ultimate product of the HPA axis, which plays a negative feedback regulating role on the HPA axis. GC can inhibit the production of inflammatory factors and autoantibodies, and antagonize the excessive response of the immune system. Its biological properties are achieved by combining with GR in tissues. Studies have shown that $5-\mathrm{HT}$, which is a neurotransmitter, has a central content that is directly proportional to the activity of HPA. 5-HT can increase the secretion of CORT by promoting $\mathrm{CRH}$ secretion in the hypothalamus, thereby producing anti-inflammatory and analgesic effects ${ }^{5}$. Therefore, the levels of GR and 5-HT receptors in the center can be used to reflect whether the functional status of HPA is normal. The pro-inflammatory Loading [MathJax]/jax/output/CommonHTML/jax.js 
factors IL-17, IL- 6 and anti-inflammatory factors TGF- $\beta$, IL-10 are important cytokines secreted by helper Th17 cells and regulatory T cells, respectively. The content of these two kinds of factors can be used to reflect the changes of autoimmune inflammation.

In this study, we observed that compared with normal rats, CRH, ACTH, CORT, L-17, and IL-6 levels in serum increased, while TGF- $\beta_{1}$ and IL-10 levels in serum decreased, and $\mathrm{GR}_{a}$ and $5-\mathrm{HT}_{1 \mathrm{~A}}$ receptor contents in spinal cord decreased in the model rats. This suggests that LDH has autoimmune imbalance, and its formation may be related to long-term hypofunction of HPA axis. Clinical observations can provide evidence for this point of view: $\triangle \mathrm{LDH}$ patients have a history of strains such as sedentary sitting or excessive waist activity before the onset of illness, and the body has chronic fatigue status, which is

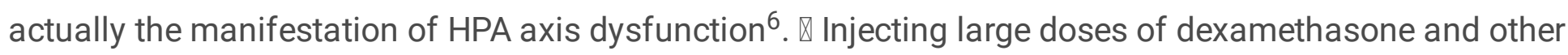
exogenous CORT drugs into the epidural of the lumbar spine is still the most effective method to alleviate LDH pain in the waist and legs ${ }^{7}$, which shows that CORT is insufficiently secreted in LDH. $\triangle$ LDH patients can usually be cured by conservative treatment, but the radiography shows no difference before and after treatment,and the lumbar disc is still protruding out of place. This shows that mechanical compression in the spinal canal is not the pathological core of LDH, and the secondary autoimmune imbalance is the key to the pathogenesis. As mentioned earlier, immune imbalance also means HPA axis function imbalance, which shows that there is a close relationship between HPA axis dysfunction and LDH.

LDH can be classified as "lumbago and leg pain" according to its clinical manifestations. "The essence of pulse" of SuWen says: "The waist is the house of the kidney. If the waist can't move, it means the kidney will be exhausted." It is clearly pointed out that the fundamental pathogenesis of low back pain is kidney deficiency. The kidney is the place where essence is stored and Qi is absorbed. It is convenient for the waist and feet. If the kidney is deficient in essence and energy is lost, the waist will be restlessly painful, and the legs and feet will be not easy to motion. As the saying goes in "Metabolic disorders of five body fluid" of LingShu: "...Kidney deficiency, so back pain and shin weak." From the perspective of clinical practice, most of the patients with LDH have the syndrome characteristics of kidney-Yang deficiency, such as fatigue, chilly limbs, poor libido, pale tongue and white fur, fine pulse, which is consistent with the purpose of The Inner Canon of Huangdi. Shen Ziyin's team ${ }^{8}$ has confirmed that the material basis of kidney-Yang-deficiency syndrome lies in the low function of hypothalamus-pituitary-target gland axis. Therefore, LDH can be induced by HPA axis dysfunction in the state of "kidney-Yang-deficiency". Chronic strain causes fatigue and dysfunction of HPA axis, followed by inadequate secretion of hormones such as GC, which leads to a sustained autoimmune inflammatory response induced by nucleus pulposus, a foreign body protruding from the spinal canal. It may be the immunological mechanism of LDH.

The waist-rubbing method used in this study originated from the therapy of lumbago contained in a Qing Dynasty medical work named Essentials of Exercise Qigong and Tuina ${ }^{9}$. The work also emphasizes the relationship between kidney deficiency and lumbago, and advocates using rubbing method to treat lumbago and leg pain. This method is simple, stable and safe. It is widely used as an end technique even in the clinical treatment of LDH by modern Tuina. Rubbing method itself has a soft warm effect, which 
can make patients get a good relaxation experience, and at the same time produce a pleasant postoperative mood, which undoubtedly promotes the functional recovery of HPA axis, which is in charge of emotional regulation. At the same time, because the waist is the house of the kidney, using rubbing method to stimulate the Shenshu, Mingmen, Yaoyangguan and other acupoints in the waist will have the effect of nourishing the kidney, warming the Yang and relieving the pain ${ }^{10}$. Therefore, the mechanism of waist-rubbing therapy for LDH should be to inhibit the autoimmunity of lumbar spinal canal by enhancing the function of HPA axis.

\section{Conclusion}

In this study, the waist-rubbing method can significantly improve the HPA axis activity of LDH model rats to promote the secretion of $\mathrm{GC}$, and increase $\mathrm{GR}_{\mathrm{a}}$ and $5-\mathrm{HT}_{1 \mathrm{~A}}$ receptors in the spinal cord, so as to effectively inhibit the autoimmune inflammatory response in the lumbar spinal canal. The model of forelimb-free standing rats can better simulate the real pathological process of LDH caused by long-term chronic strain. This suggests that there is an autoimmune imbalance in $\mathrm{LDH}$, which may be related to the long-term dysfunction of HPA axis. The waist-rubbing method may eliminate the autoimmune inflammatory reaction of LDH by correcting the hypofunction of HPA axis, so as to achieve the therapeutic effect of LDH.

\section{Abbreviations}

LDH

Lumbar disc herniation

HPA

Hypothalamic-pituitary-adrenal axis

$\mathrm{CRH}$

Corticotropin releasing hormone

ACTH

Adrenocorticotropic hormone

CORT

Cortisol

IL-17

Interleukin-17

IL-6

Interleukin-6

TGF- $\beta_{1}$

Transforming growth factor- $\beta 1$

IL-10

Interleukin-10

GR

Loading [MathJax]/jax/output/CommonHTML/jax.js 
Glucocorticoid receptora

$5-\mathrm{HT}_{1 \mathrm{~A}}$

Five hydroxytryptamine $1 \mathrm{~A}$ receptor

ELISA

Enzyme-linked immunosorbent assay

GC

Glucocorticoid

\section{Declarations}

\section{Ethics declarations}

The protocols were approved by the Ethics Committee of the Nanjing University of Chinese Medicine (No. 20190508).

\section{Consent for publication}

The manuscript is approved by all authors for publication.

\section{Availability of data and materials}

The research data generated from this study is included within the article.

\section{Competing interests}

The authors declare that they have no competing interests.

\section{Funding}

This work was supported by Youth program of Jiangsu Natural Science Foundation, China (No. BK20171070).

\section{Contributions}

YHQ designed the study. YHQ and YX and GHS participated in the experiment. YHQ conducted the analysis of variance $L S D$ - $t$ test and draft the manuscript. $Y C W$ and $Y X$ supervised the study and provided the technical support and advices for the study. All authors read and approved the final manuscript.

\section{Acknowledgements}

No applicable.

\section{References}


1. Shi XM, Jin YC. Encyclopedia of Chinese Tuina. China: Hunan Science and Technology Press; 2018. p. 578.

2. Qin $Y H$, Wu YC, Xiong $Y$, et al. Clinical observation on the treatment of lumbar disc herniation based on the theory of "the waist is the house of the kidney" by rubbing the waist and pressing the points. World science technology-modernization of traditional Chinese medicine materia medica. 2018;20(10):1854-9.

3. Liu D. Establishment of the rat model of lumbar disc degeneration induced by removing two forelimbs and Study on the intervention mechanism of Gubi mixture. Yunnan University of traditional Chinese medicine, 2014.

4. Naylor A, Happey F, Tumer RL, et al. Enzymic and immunological activity in the intervertebral disk. Orthop clin North Am. 1975;6(1):51-8.

5. Wu XF, Zheng XN, Guo X, et al. Research progress of acupuncture on 5-HT and its receptor, HPA axis related hormones in insomnia rats. Chinese archives of traditional Chinese medicine. 2018;36(01):127-9.

6. Wang J, Kuang SX. Research progress of correlation between immune system and HPA axis. Journal of Hubei University of traditional Chinese medicine. 2017;19(02):110-4.

7. Wang JN, Fu ZJ, Zhao XL, et al. Retrospective analysis of the efficacy of epidural injection of glucocorticoid in the treatment of lumbar disc herniation. Chinese Journal of pain medicine. 2012;18(02):78-80.

8. Huang JH, Shen ZY, Cai WJ, et al. The progress of hypothalamus pituitary adrenocortical axis programming and its significance to 'Syndrome' research. Chinese Journal of traditional Chinese medicine pharmacy. 2013;28(12):3620-3.

9. [Qing] Wang QX, Wang QS Essentials of Exercise Qigong and Tuina, Traditional Chinese Medicine Classics Press, 1986: 8.

10. Liu MJ, Sun WQ. Tuina. People's Health Press, 2016: 101. 